\title{
Synthesis of Tetrasubstituted Olefins by Pd-Catalyzed Addition of Arylboronic Acids to Internal Alkynes
}

\author{
Chengxiang Zhou and Richard C. Larock* \\ Department of Chemistry, Iowa State University, Ames, Iowa 50011 \\ larock@iastate.edu
}

\section{General}

The ${ }^{1} \mathrm{H}$ and ${ }^{13} \mathrm{C}$ NMR spectra were recorded at $300 \mathrm{MHz}$ or $400 \mathrm{MHz}$ and $75 \mathrm{MHz}$ or $100 \mathrm{MHz}$, respectively. The COESY and NOESY NMR spectra were recorded at 400 MHz. Thin layer chromatography was performed using commercially prepared 60-mesh silica gel plates (Whatman K6F), and visualization was effected with short wavelength UV light $(254 \mathrm{~nm})$. Low resolution mass spectra were recorded on a Finnigan TSQ700 triple quadruple mass spectrometer (Finnigan MAT, San Jose, CA). High resolution mass spectra were recorded on a Kratos MS50TC double focusing magnetic sector mass spectrometer using EI at $70 \mathrm{eV}$. Anhydrous forms of DMSO, ethyl ether, hexanes, ethyl acetate, and molecular sieves were purchased from Fisher Scientific Co. All palladium salts were donated by Johnson Matthey Inc. and Kawaken Fine Chemicals Co., Ltd. All arylboronic acids were donated by Frontier Scientific Co., Ltd.

\section{General procedure for the synthesis of tetrasubstituted olefins (Table 2)}

$\mathrm{Pd}(\mathrm{OAc})_{2}(0.0125 \mathrm{mmol})$, the internal alkyne $(0.25 \mathrm{mmol})$, the arylboronic acid $(1.25$ mmol), molecular sieves ( $4 \AA, 0.2 \mathrm{~g})$, and DMSO $(2 \mathrm{~mL})$ were placed in a 6-dram vial. The vial was sealed with a septum, degassed, and equipped with $\mathrm{O}_{2}$ balloon. The contents were stirred at room temperature or at $50{ }^{\circ} \mathrm{C}$ for $24 \mathrm{~h}$. The reaction mixture was quenched with $20 \mathrm{~mL}$ of satd $\mathrm{NaCl}$ solution. The resulting mixture was extracted three times with ethyl ether (30 mL each time). The combined organic layers were dried over anhydrous $\mathrm{MgSO}_{4}$ and the solvent was evaporated under reduced pressure. The product was isolated by chromatography on a silica gel column.

\section{Characterization data:}

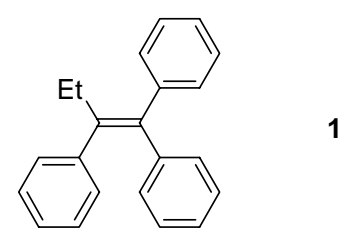

1,1,2-Triphenyl-1-butene (1). White solid: $\mathrm{mp} \mathrm{80-82}{ }^{\circ} \mathrm{C}$ (lit. ${ }^{1} \mathrm{mp} 80-81{ }^{\circ} \mathrm{C}$ ); ${ }^{1} \mathrm{H} \mathrm{NMR}$ $\left(400 \mathrm{MHz}, \mathrm{CDCl}_{3}\right) \delta 0.96(\mathrm{t}, J=7.6 \mathrm{~Hz}, 3 \mathrm{H}), 2.50(\mathrm{q}, J=7.6 \mathrm{~Hz}, 2 \mathrm{H}), 6.88-6.93(\mathrm{~m}$, $2 \mathrm{H}), 7.00-7.08(\mathrm{~m}, 3 \mathrm{H}), 7.12-7.20(\mathrm{~m}, 5 \mathrm{H}), 7.25-7.39(\mathrm{~m}, 5 \mathrm{H}) ;{ }^{13} \mathrm{C}$ NMR $(100 \mathrm{MHz}$, $\left.\mathrm{CDCl}_{3}\right) \delta 13.88,29.27,125.96,126.39,126.84,127.59,128.04,128.38,129.71,129.93$, 
131.01, 139.02, 142.39, 142.44, 143.24, 143.74; IR $\left(\mathrm{CDCl}_{3}, \mathrm{~cm}^{-1}\right)$ 3023, 2959, 2927, 2858, 1598, 1490; MS (EI) m/z (rel intensity) $284\left(\mathrm{M}^{+}, 100\right), 269$ (26); HRMS (EI) calcd for $\mathrm{C}_{22} \mathrm{H}_{20}$ 284.1565, found: 284.1572 .

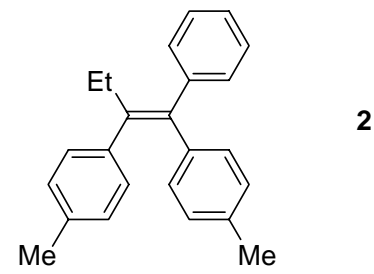

(Z)-1,2-Di-(4-methylphenyl)-1-phenyl-1-butene (2). Colorless oil; ${ }^{1} \mathrm{H}$ NMR (300 $\left.\mathrm{MHz}, \mathrm{CDCl}_{3}\right) \delta 0.95(\mathrm{t}, J=7.5 \mathrm{~Hz}, 3 \mathrm{H}), 2.22(\mathrm{~s}, 3 \mathrm{H}), 2.31(\mathrm{~s}, 3 \mathrm{H}), 2.47(\mathrm{q}, J=7.5 \mathrm{~Hz}$, $2 \mathrm{H}), 6.80-6.86(\mathrm{~m}, 4 \mathrm{H}), 7.00-7.07(\mathrm{~m}, 4 \mathrm{H}), 7.26-7.36(\mathrm{~m}, 5 \mathrm{H}) ;{ }^{13} \mathrm{C} \mathrm{NMR}(75 \mathrm{MHz}$, $\left.\mathrm{CDCl}_{3}\right) \delta 13.90,21.35,21.43,29.31,126.67,128.34,128.36,128.83,129.73,129.78$, $130.89,135.35,135.79,138.54,139.49,140.47,141.80,144.25 ;$ IR $\left(\mathrm{CDCl}_{3}, \mathrm{~cm}^{-1}\right) 3022$, 2958, 2927, 2860, 1598, 1492; MS (EI) m/z (rel intensity) $312\left(\mathrm{M}^{+}, 100\right), 297$ (23); HRMS (EI) calcd for $\mathrm{C}_{24} \mathrm{H}_{24} 312.1878$, found: 312.1886 .

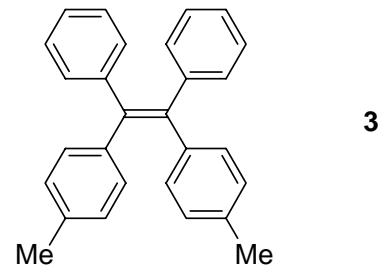

(Z)-1,2-Di-(4-methylphenyl)-1,2-diphenylethene (3). ${ }^{2}$ White solid: mp $143-145{ }^{\circ} \mathrm{C} ;{ }^{1} \mathrm{H}$ NMR $\left(400 \mathrm{MHz}, \mathrm{CDCl}_{3}\right) \delta 2.29(\mathrm{~s}, 6 \mathrm{H}), 6.92-7.00(\mathrm{~m}, 8 \mathrm{H}), 7.03-7.06(\mathrm{~m}, 4 \mathrm{H}), 7.09-7.12$ $(\mathrm{m}, 6 \mathrm{H}) ;{ }^{13} \mathrm{C} \mathrm{NMR}\left(100 \mathrm{MHz}, \mathrm{CDCl}_{3}\right) \delta 21.52,126.45,127.81,128.65,131.43,131.63$, 136.16, 140.62, 141.17, 144.39; IR $\left(\mathrm{CDCl}_{3}, \mathrm{~cm}^{-1}\right)$ 3050, 3022, 2920, 2860, 1509, 1443; MS (EI) m/z (rel intensity) $360\left(\mathrm{M}^{+}, 100\right), 345$ (22); HRMS (EI) calcd for $\mathrm{C}_{28} \mathrm{H}_{24}$ 360.1878 , found: 360.1883 .

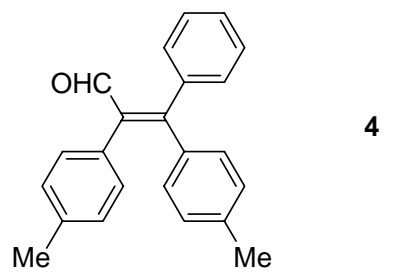

(E)-2,3-Di-(4-methylphenyl)-3-phenylpropenal (4). Light yellow solid: mp 145-146 ${ }^{\circ} \mathrm{C} ;{ }^{1} \mathrm{H}$ NMR $\left(400 \mathrm{MHz}, \mathrm{CDCl}_{3}\right) \delta 2.28(\mathrm{~s}, 3 \mathrm{H}), 2.31$ (s, 3H), 6.87-6.90 (m, 2H), 6.937.00 (m, 4H), 7.04-7.07 (m, 2H), 7.27-7.31 (m, 2H), 7.41-7.45 (m, 3H), 9.68 (s, $1 \mathrm{H}) ;{ }^{13} \mathrm{C}$ NMR (100 MHz, $\left.\mathrm{CDCl}_{3}\right) \delta 21.59,21.61,128.41,128.80,129.13,129.74,131.29,131.40$, 131.94, 133.52, 137.35, 138.25, 139.08, 139.67, 139.69, 160.55, 194.15; IR ( $\mathrm{CDCl}_{3}, \mathrm{~cm}^{-}$ $\left.{ }^{1}\right) 3052,3025,2919,2852,2720,1669,1508,1443 ; \mathrm{MS}(\mathrm{EI}) \mathrm{m} / \mathrm{z}$ (rel intensity) $312\left(\mathrm{M}^{+}\right.$, 100), 297 (29), 252 (13); HRMS (EI) calcd for $\mathrm{C}_{23} \mathrm{H}_{20} \mathrm{O} 312.1514$, found: 312.1520. 


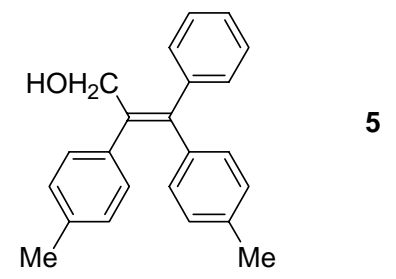

(E)-2,3-Di-(4-methylphenyl)-3-phenyl-2-propen-1-ol (5). White solid: mp 93-96 ${ }^{\circ} \mathrm{C}$; ${ }^{1} \mathrm{H}$ NMR $\left(400 \mathrm{MHz}, \mathrm{CDCl}_{3}\right) \delta 1.27(\mathrm{~s}, 1 \mathrm{H}), 2.22(\mathrm{~s}, 3 \mathrm{H}), 2.29(\mathrm{~s}, 3 \mathrm{H}), 4.45(\mathrm{~s}, 2 \mathrm{H}), 6.81$ $(\mathrm{d}, J=8.0 \mathrm{~Hz}, 2 \mathrm{H}), 6.86(\mathrm{~m}, J=8.0 \mathrm{~Hz}, 2 \mathrm{H}), 7.02(\mathrm{~d}, J=8.0 \mathrm{~Hz}, 2 \mathrm{H}), 7.11(\mathrm{~d}, J=8.0$ $\mathrm{Hz}, 2 \mathrm{H}), 7.29-7.38(\mathrm{~m}, 5 \mathrm{H}) ;{ }^{13} \mathrm{C} \mathrm{NMR}\left(100 \mathrm{MHz}, \mathrm{CDCl}_{3}\right) \delta 21.41,21.46,65.43,127.39$, $128.46,128.54,129.29$, 129.91, 129.96, 130.80, 136.29, 136.69, 137.43, 138.03, 139.59, 142.46, 142.83; IR $\left(\mathrm{CDCl}_{3}, \mathrm{~cm}^{-1}\right) 3597,3053,2986,2925,2854,1509,1443 ; \mathrm{MS}(\mathrm{EI})$ $\mathrm{m} / \mathrm{z}$ (rel intensity) $314\left(\mathrm{M}^{+}, 100\right), 266$ (14), 252 (15); HRMS (EI) calcd for $\mathrm{C}_{23} \mathrm{H}_{22} \mathrm{O}$ 314.1671, found: 314.1676 .

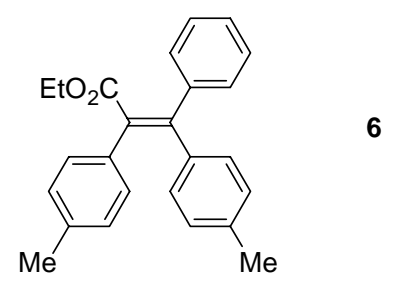

Ethyl (E)-2,3-di-(4-methylphenyl)-3-phenylpropenoate (6). White solid: mp 127-129 ${ }^{\circ} \mathrm{C} ;{ }^{1} \mathrm{H}$ NMR $\left(300 \mathrm{MHz}, \mathrm{CDCl}_{3}\right) \delta 0.96(\mathrm{t}, J=7.2 \mathrm{~Hz}, 3 \mathrm{H}), 2.28(\mathrm{~s}, 3 \mathrm{H}), 2.30(\mathrm{~s}, 3 \mathrm{H})$, 4.01 (q, $J=7.2 \mathrm{~Hz}, 2 \mathrm{H}), 6.89-7.03(\mathrm{~m}, 8 \mathrm{H}), 7.28-7.33(\mathrm{~m}, 5 \mathrm{H}) ;{ }^{13} \mathrm{C}$ NMR $(75 \mathrm{MHz}$, $\left.\mathrm{CDCl}_{3}\right) \delta 13.94,21.49,21.52,61.06,128.28,128.49,128.80,129.20,129.44,129.95$, 131.11, 133.48, 135.00, 137.29, 137.63, 138.04, 143.15, 145.59, 171.09; IR $\left(\mathrm{CDCl}_{3}, \mathrm{~cm}^{-}\right.$ $\left.{ }^{1}\right) 3053,3024,2918,2871,1715,1509,1445 ; \mathrm{MS}(\mathrm{EI}) \mathrm{m} / \mathrm{z}$ (rel intensity) $356\left(\mathrm{M}^{+}, 100\right)$, 282 (22), 266 (33); HRMS (EI) calcd for $\mathrm{C}_{25} \mathrm{H}_{24} \mathrm{O}_{2} 356.1776$, found: 356.1781 .

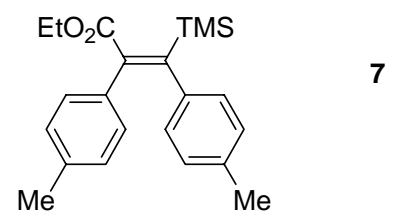

Ethyl (E)-2,3-di-(4-methylphenyl)-3-(trimethylsilyl)propenoate (7). Colorless oil; ${ }^{1} \mathrm{H}$ NMR $\left(400 \mathrm{MHz}, \mathrm{CDCl}_{3}\right) \delta 0.13(\mathrm{~s}, 9 \mathrm{H}), 1.30(\mathrm{t}, J=7.2 \mathrm{~Hz}, 3 \mathrm{H}), 2.20(\mathrm{~s}, 3 \mathrm{H}), 2.23(\mathrm{~s}$, $3 \mathrm{H}), 4.23(\mathrm{q}, J=7.2 \mathrm{~Hz}, 2 \mathrm{H}), 6.71(\mathrm{~d}, J=8.0 \mathrm{~Hz}, 2 \mathrm{H}), 6.88-6.96(\mathrm{~m}, 6 \mathrm{H}) ;{ }^{13} \mathrm{C} \mathrm{NMR}$ $\left(100 \mathrm{MHz}, \mathrm{CDCl}_{3}\right) \delta 0.01,14.28,21.26,21.33,61.24,127.80,128.33,128.47,129.30$, 134.63, 134.80, 136.45, 139.37, 144.05, 151.39, 169.98; IR $\left(\mathrm{CDCl}_{3}, \mathrm{~cm}^{-1}\right)$ 3020, 2980, 2922, 1716, 1506, 1442; MS (EI) m/z (rel intensity) $352\left(\mathrm{M}^{+}, 18\right), 337$ (100), 309 (30); HRMS (EI) calcd for $\mathrm{C}_{22} \mathrm{H}_{28} \mathrm{SiO}_{2} 352.1859$, found: 352.1865 . 


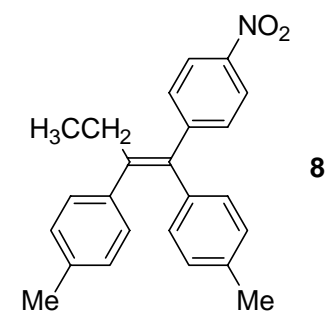

(E)-1,2-Di-(4-methylphenyl)-1-(4-nitrophenyl)-1-butene (8). Yellow solid: mp 136$138{ }^{\circ} \mathrm{C} ; \quad{ }^{1} \mathrm{H}$ NMR $\left(400 \mathrm{MHz}, \mathrm{CDCl}_{3}\right) \delta 0.95(\mathrm{t}, J=7.2 \mathrm{~Hz}, 3 \mathrm{H}), 2.20(\mathrm{~s}, 3 \mathrm{H}), 2.28$ (s, $3 \mathrm{H}), 2.43(\mathrm{q}, J=7.2 \mathrm{~Hz}, 2 \mathrm{H}), 6.73(\mathrm{~d}, J=8.0 \mathrm{~Hz}, 2 \mathrm{H}), 6.85(\mathrm{~d}, J=8.0 \mathrm{~Hz}, 2 \mathrm{H}), 7.00-$ $7.03(\mathrm{~m}, 4 \mathrm{H}), 7.40(\mathrm{~d}, J=8.0 \mathrm{~Hz}, 2 \mathrm{H}), 8.20(\mathrm{~d}, J=8.0 \mathrm{~Hz}, 2 \mathrm{H}) ;{ }^{13} \mathrm{C}$ NMR $(100 \mathrm{MHz}$, $\left.\mathrm{CDCl}_{3}\right) \delta 13.84,21.38,21.47,29.31,123.77,128.72,128.99,129.57,130.65,130.86$, 136.18, 136.48, 136.66, 138.44, 139.22, 143.96, 146.66, 151.29; IR $\left(\mathrm{CDCl}_{3}, \mathrm{~cm}^{-1}\right) 3057$, 3022, 2970, 2932, 1516, 1442; MS (EI) m/z (rel intensity) 357 ( $\left.{ }^{+}, 100\right), 342$ (19); HRMS (EI) calcd for $\mathrm{C}_{24} \mathrm{H}_{23} \mathrm{NO}_{2} 357.1729$, found: 357.1736 .

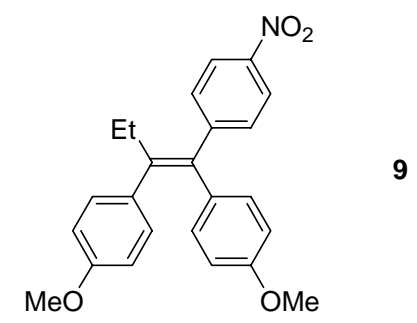

(E)-1,2-Di-(4-methoxyphenyl)-1-(4-nitrophenyl)-1-butene (9). Yellow oil; ${ }^{1} \mathrm{H}$ NMR $\left(400 \mathrm{MHz}, \mathrm{CDCl}_{3}\right) \delta 0.95$ (t, J=7.2 Hz, 3H), 2.42 (q, J=7.2 Hz, 2H), 3.70 (s, $\left.3 \mathrm{H}\right), 3.77$ (s, 3H), 6.59 (d, $J=7.6 \mathrm{~Hz}, 2 \mathrm{H}), 6.72-6.76(\mathrm{~m}, 4 \mathrm{H}), 7.04(\mathrm{~d}, J=8.4 \mathrm{~Hz}, 2 \mathrm{H}), 7.38$ (d, $J=$ $8.4 \mathrm{~Hz}, 2 \mathrm{H}), 8.20(\mathrm{~d}, J=8.4 \mathrm{~Hz}, 2 \mathrm{H}) ;{ }^{13} \mathrm{C} \mathrm{NMR}\left(100 \mathrm{MHz}, \mathrm{CDCl}_{3}\right) \delta 13.88,29.17$, $55.31,55.37,113.39,113.68,123.76,130.65,130.85,132.20,133.70,134.69,136.10$, 143.23, 146.61, 151.41, 158.03, 158.40; IR $\left(\mathrm{CDCl}_{3}, \mathrm{~cm}^{-1}\right)$ 3033, 2963, 2932, 2836, 1605, 1509, 1441; MS (EI) m/z (rel intensity) $389\left(\mathrm{M}^{+}, 100\right), 374$ (20); HRMS (EI) calcd for $\mathrm{C}_{24} \mathrm{H}_{23} \mathrm{NO}_{4} 389.1627$, found: 389.1634 .

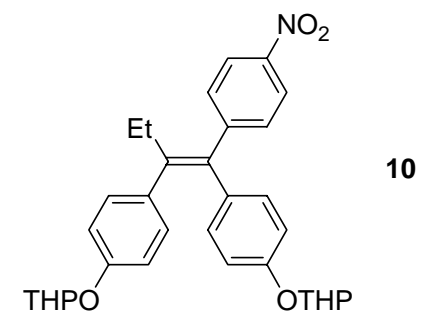

(Z)-1,2-Di-[4-tetrahydropyranyl-2-oxy)phenyl]-1-(4-nitrophenyl)-1-butene

(10). Yellow oil; ${ }^{1} \mathrm{H}$ NMR $\left(400 \mathrm{MHz}, \mathrm{CDCl}_{3}\right) \delta 0.92(\mathrm{t}, J=7.6 \mathrm{~Hz}, 3 \mathrm{H}), 1.57-1.69(\mathrm{~m}, 6 \mathrm{H})$, $1.78-1.97$ (m, 6H), 2.43 (q, $J=7.6 \mathrm{~Hz}, 2 \mathrm{H}), 3.52-3.62(\mathrm{~m}, 2 \mathrm{H}), 3.82-3.98(\mathrm{~m}, 2 \mathrm{H}), 5.26-$ $5.28(\mathrm{~m}, 1 \mathrm{H}), 5.35-5.37(\mathrm{~m}, 1 \mathrm{H}), 6.70-6.75(\mathrm{~m}, 4 \mathrm{H}), 6.86(\mathrm{~d}, J=8.8 \mathrm{~Hz}, 2 \mathrm{H}), 7.02(\mathrm{~d}, J=$ $8.8 \mathrm{~Hz}, 2 \mathrm{H}), 7.38(\mathrm{~d}, J=8.8 \mathrm{~Hz}, 2 \mathrm{H}), 8.19(\mathrm{~d}, J=8.8 \mathrm{~Hz}, 2 \mathrm{H}) ;{ }^{13} \mathrm{C}$ NMR $(100 \mathrm{MHz}$, $\left.\mathrm{CDCl}_{3}\right) \delta 13.89,19.19,19.27,25.40,25.45,29.24,30.62,30.70,62.52,62.59,96.60$, $96.74,115.80,116.13,123.72,130.67,130.76,132.11,134.57,135.46,136.174,143.31$, 146.59, 151.39, 155.61, 155.98; IR $\left(\mathrm{CDCl}_{3}, \mathrm{~cm}^{-1}\right) 3332,2948,2871,1702,1608,1512$, 
1440; MS (EI) m/z (rel intensity) $529\left(\mathrm{M}^{+}, 25\right), 445$ (100); HRMS (EI) calcd for $\mathrm{C}_{32} \mathrm{H}_{35} \mathrm{NO}_{6}$ 529.2464, found: 529.2474 .

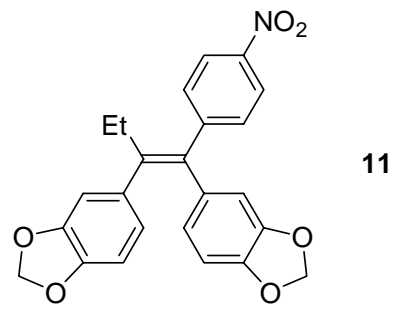

(Z)-1,2-Di-(3,4-methylenedioxyphenyl)-1-(4-nitrophenyl)-1-butene (11). Yellow solid: mp 127-129 ${ }^{\circ} \mathrm{C}$; ${ }^{1} \mathrm{H}$ NMR $\left(400 \mathrm{MHz}, \mathrm{CDCl}_{3}\right) \delta 0.94(\mathrm{t}, J=7.2 \mathrm{~Hz}, 3 \mathrm{H}), 2.36(\mathrm{q}, J=7.2$ $\mathrm{Hz}, 2 \mathrm{H}), 5.85$ (s, 2H), $5.92(\mathrm{~s}, 2 \mathrm{H}), 6.30-6.33(\mathrm{~m}, 2 \mathrm{H}), 6.52-6.67$ (m, 4H), 7.36 (d, $J=8.8$ $\mathrm{Hz}, 2 \mathrm{H}), 8.20(\mathrm{~d}, J=8.8 \mathrm{~Hz}, 2 \mathrm{H}) ;{ }^{13} \mathrm{C}$ NMR $\left(100 \mathrm{MHz}, \mathrm{CDCl}_{3}\right) \delta 13.79,29.36,101.12$, 101.16, 108.06, 108.35, 109.86, 111.14, 123.24, 123.80, 124.76, 130.49, 135.23, 136.02, 136.47, 143.71, 146.22, 146.48, 146.72, 147.33, 147.62, 150.83; IR $\left(\mathrm{CDCl}_{3}, \mathrm{~cm}^{-1}\right) 3072$, 2971, 2895, 1593, 1516, 1435; MS (EI) m/z (rel intensity) 417 ( $\left.{ }^{+}, 100\right), 356$ (23); HRMS (EI) calcd for $\mathrm{C}_{24} \mathrm{H}_{19} \mathrm{NO}_{6} 417.1212$, found: 417.1217 .

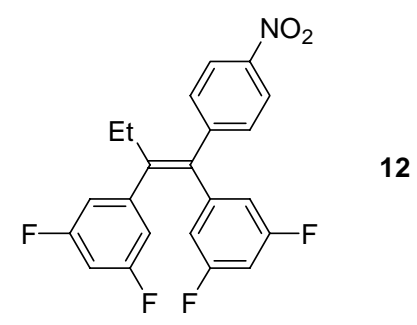

(Z)-1,2-Di-(3,5-difluorophenyl)-1-(4-nitrophenyl)-1-butene (12). Yellow oil; ${ }^{1} \mathrm{H}$ NMR $\left(400 \mathrm{MHz}, \mathrm{CDCl}_{3}\right) \delta 0.96(\mathrm{t}, J=7.2 \mathrm{~Hz}, 3 \mathrm{H}), 2.40(\mathrm{q}, J=7.2 \mathrm{~Hz}, 2 \mathrm{H}), 6.37-6.40(\mathrm{~m}$, $2 \mathrm{H}), 6.56-6.68(\mathrm{~m}, 4 \mathrm{H}), 7.38(\mathrm{~d}, J=8.4 \mathrm{~Hz}, 2 \mathrm{H}), 8.26(\mathrm{~d}, J=8.8 \mathrm{~Hz}, 2 \mathrm{H}) ;{ }^{13} \mathrm{C} \mathrm{NMR}$ $\left(100 \mathrm{MHz}, \mathrm{CDCl}_{3}\right) \delta 13.45,29.25,102.84,103.01,103.09,103.26,103.36,103.51$, $112.21,112.28,112.39,112.46,113.37,113.44,113.55,113.62,115.57,123.98,124.16$, $129.49,130.36,136.52,144.05,144.18,144.21,144.27,147.33,148.24,161.47,161.60$, 161.82, 161.95, 163.95, 164.08, 164.29, 164.42; IR $\left(\mathrm{CDCl}_{3}, \mathrm{~cm}^{-1}\right) 3050,3025,2968$, 2871, 1590, 1516, 1442; MS (EI) m/z (rel intensity) $312\left(\mathrm{M}^{+}, 61\right), 269$ (60), 191 (100); HRMS (EI) calcd for $\mathrm{C}_{22} \mathrm{H}_{15} \mathrm{NO}_{2} \mathrm{~F}_{4} 401.1039$, found: 401.1046 .

\section{Reference}

1. Okamoto, Y.; Yoshikawa, Y.; Hayashi, T. J. Organomet. Chem. 1989, 359, 143.

2. Oda, H.; Morishita, M.; Fugami, K.; Sano, H.; Kosugi, M. Chem. Lett. 1996, 9, 811. 

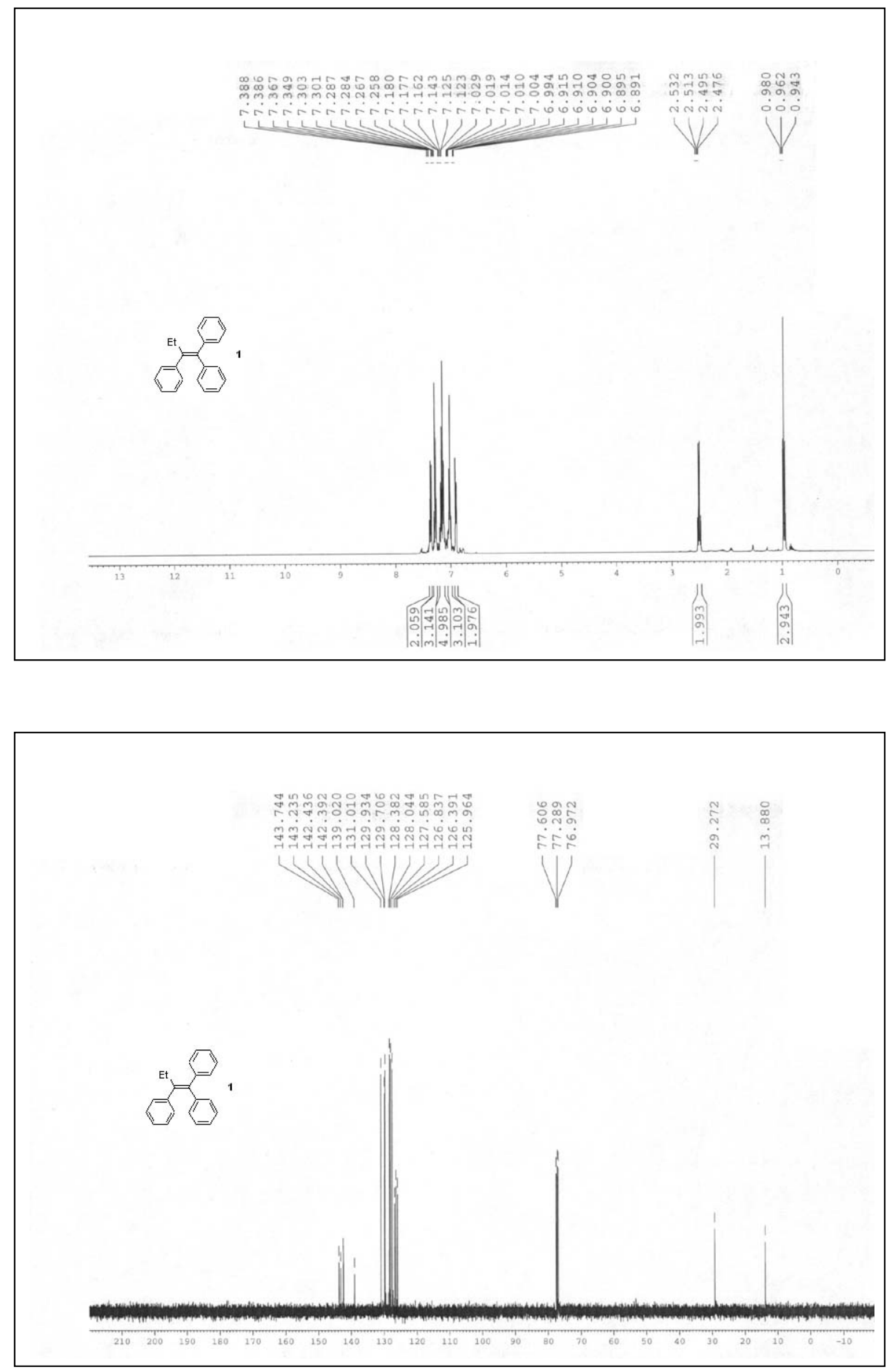

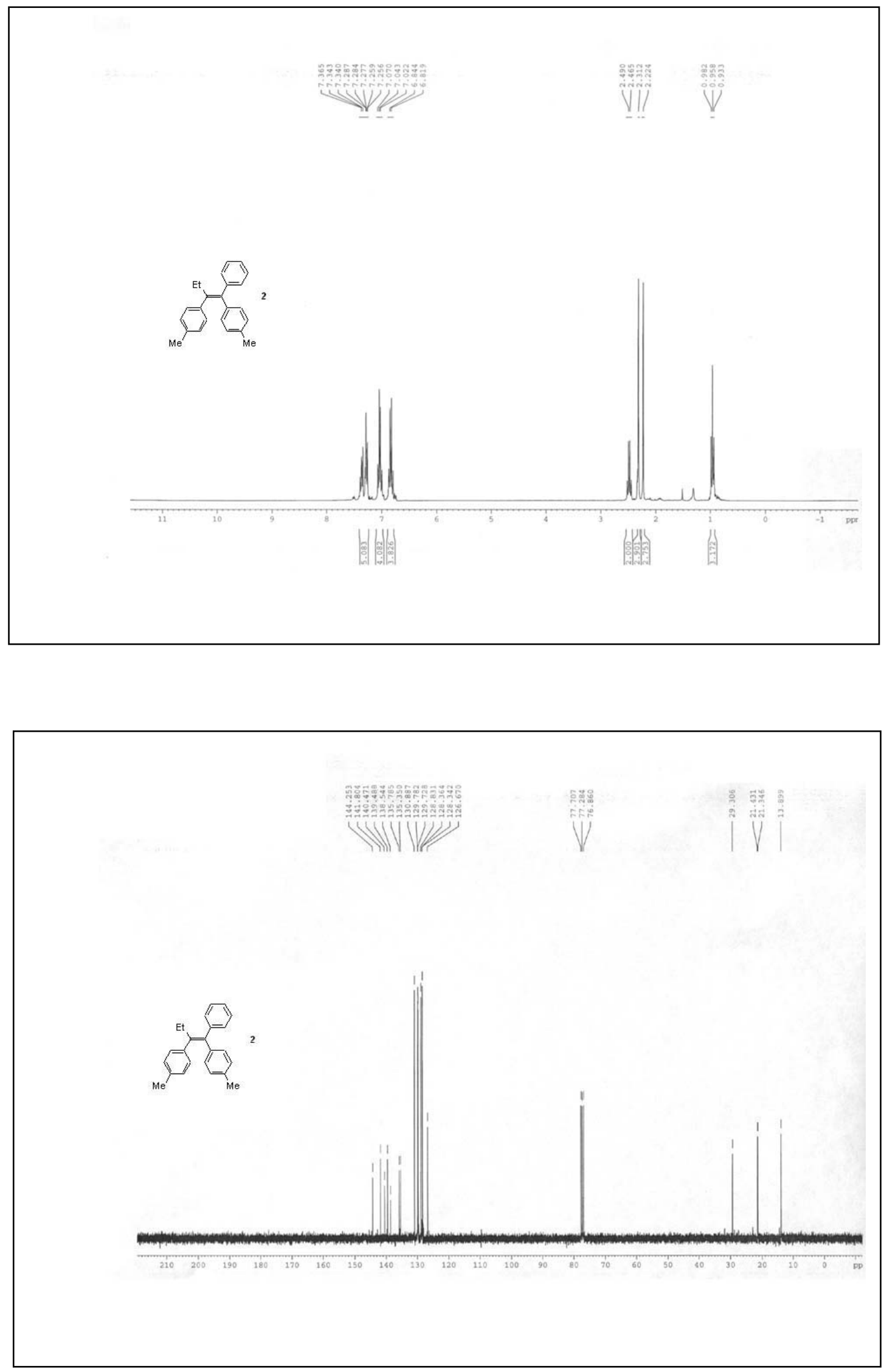

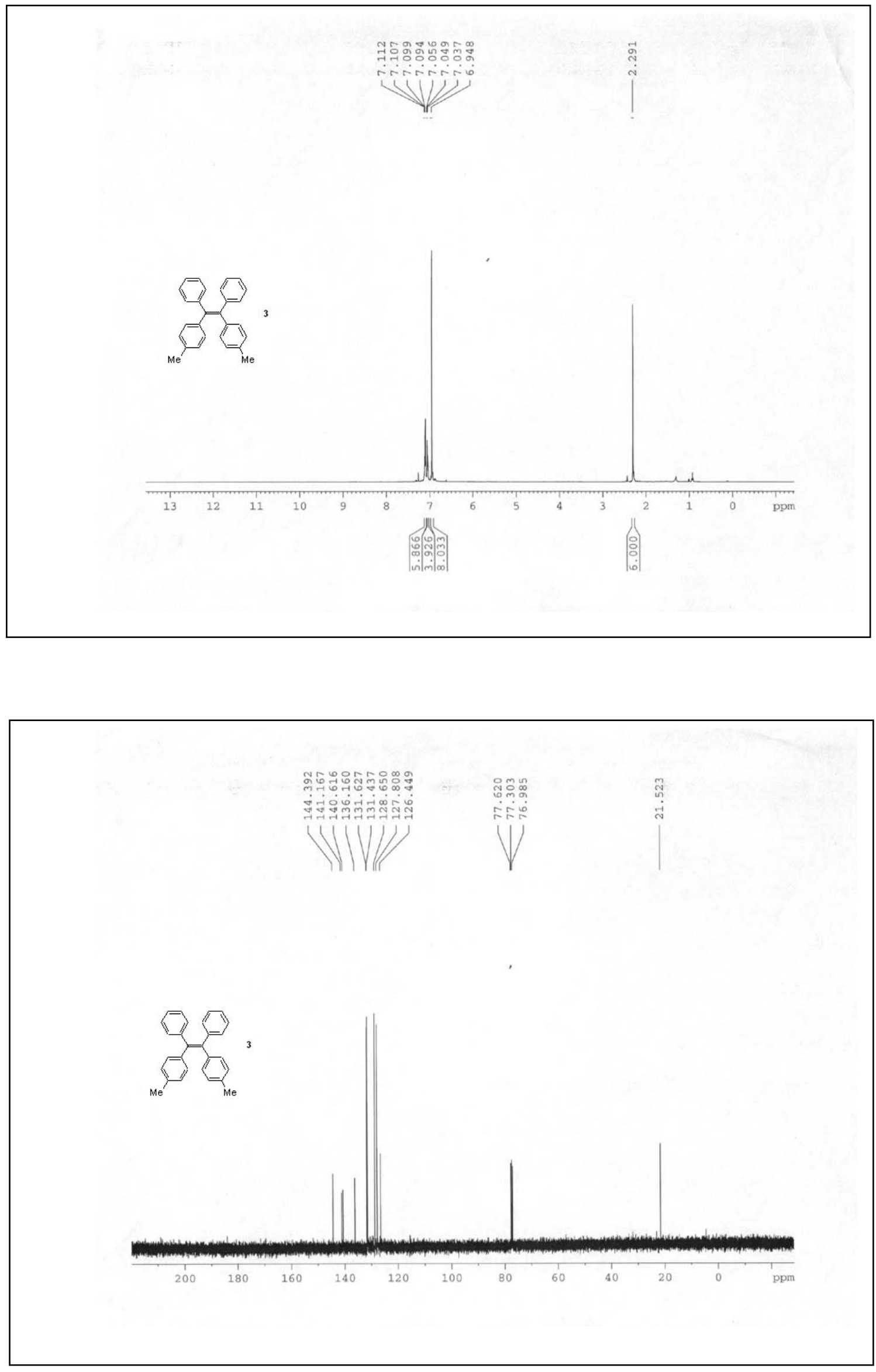

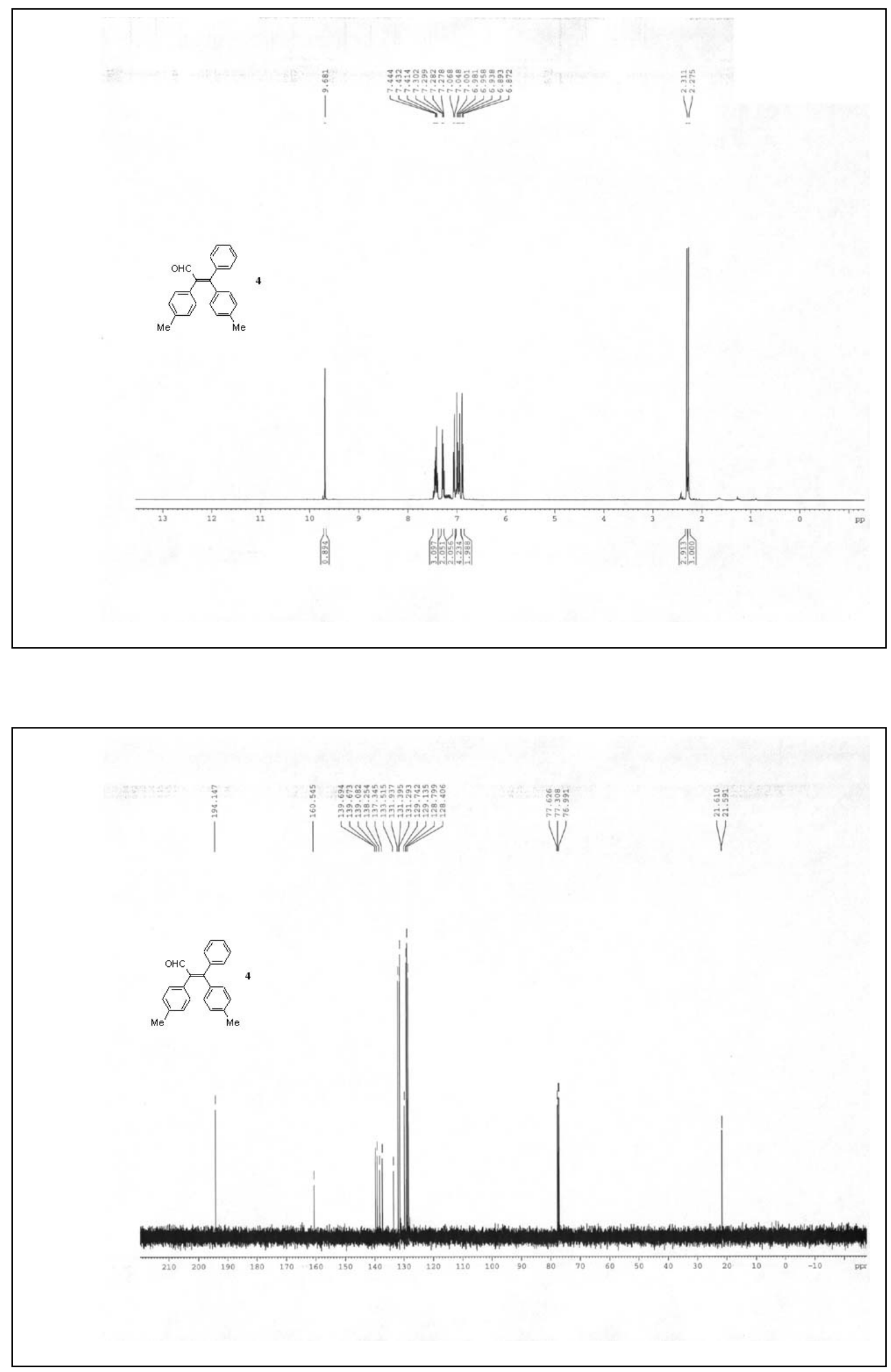

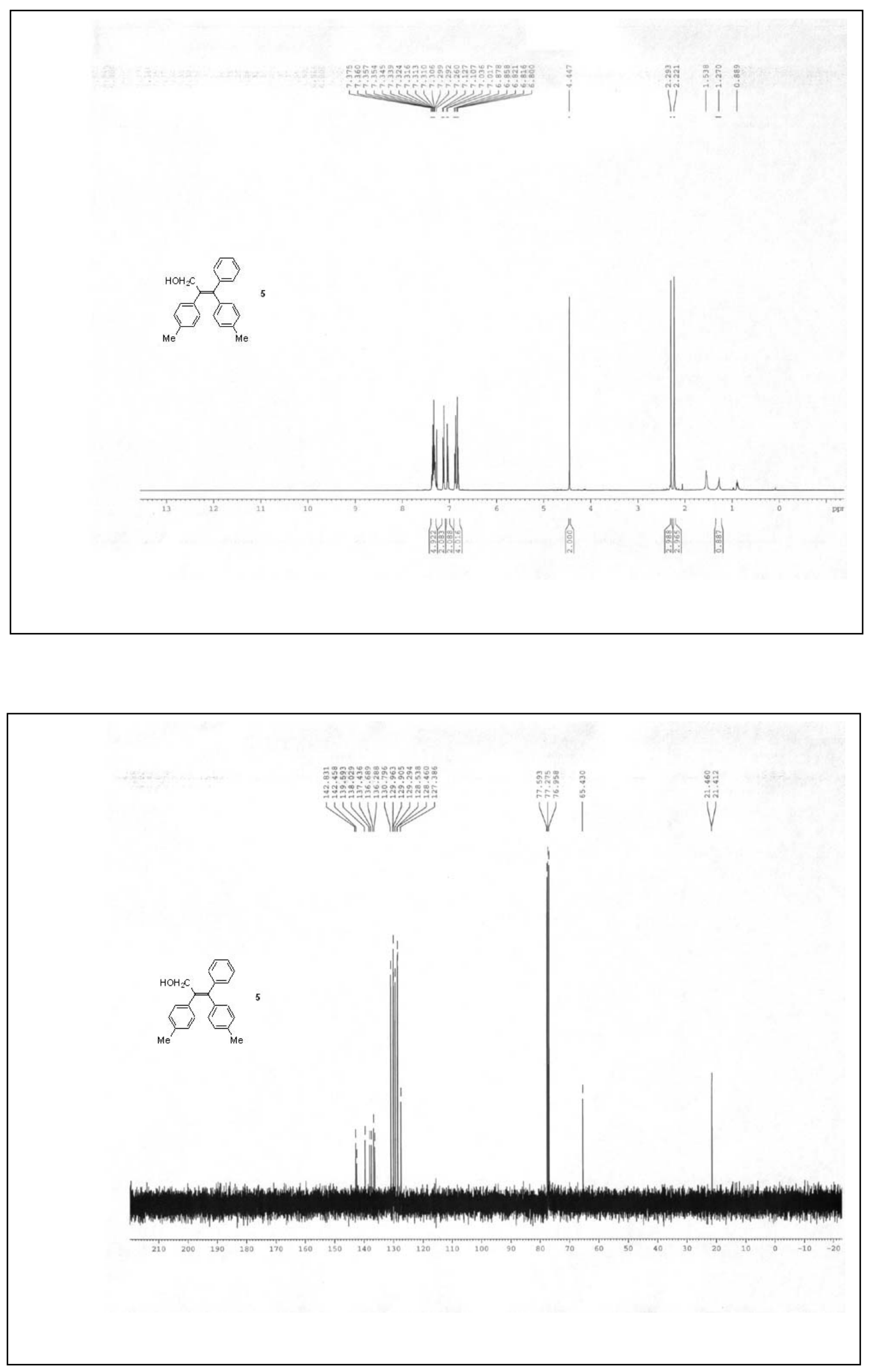

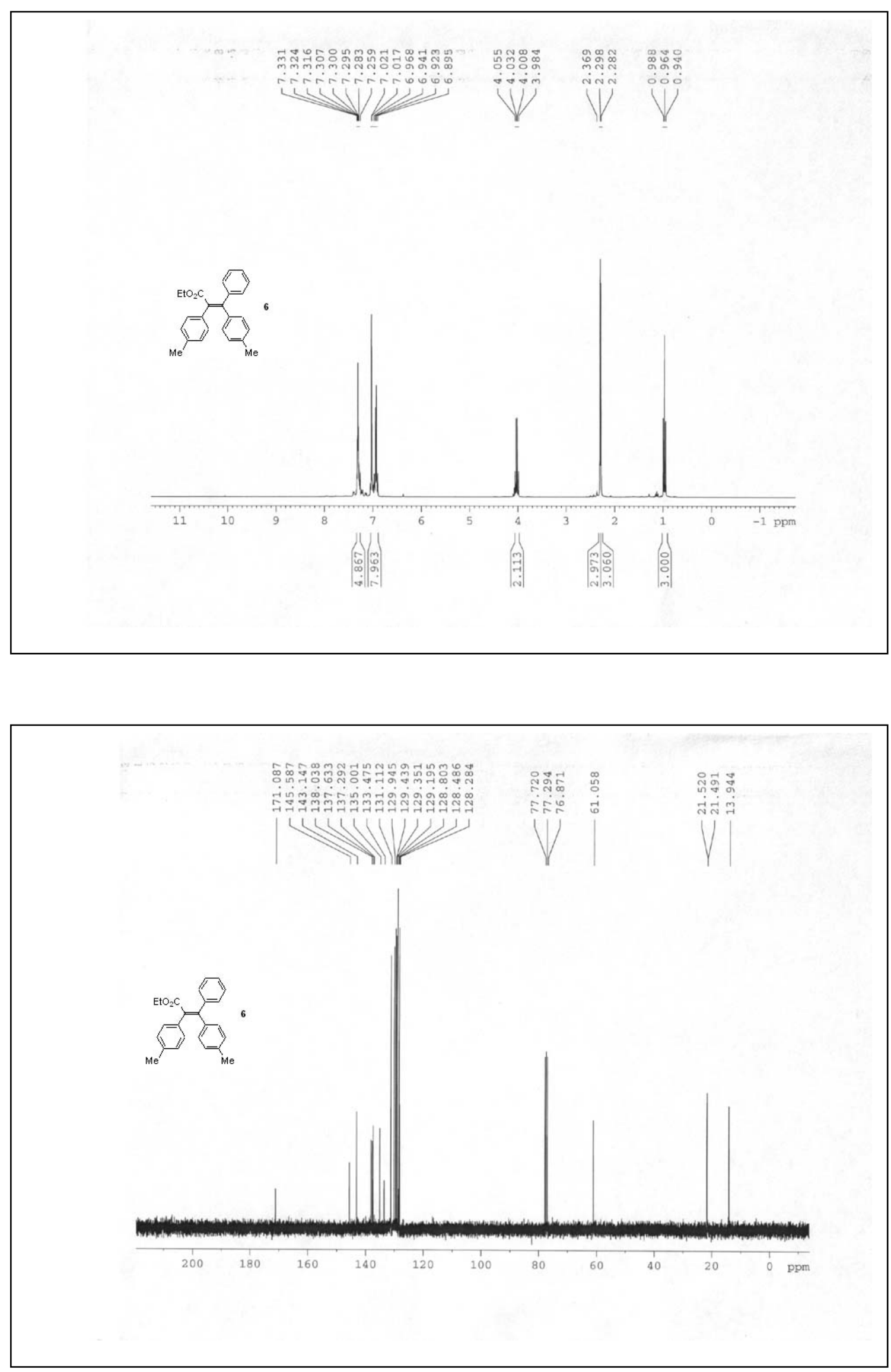

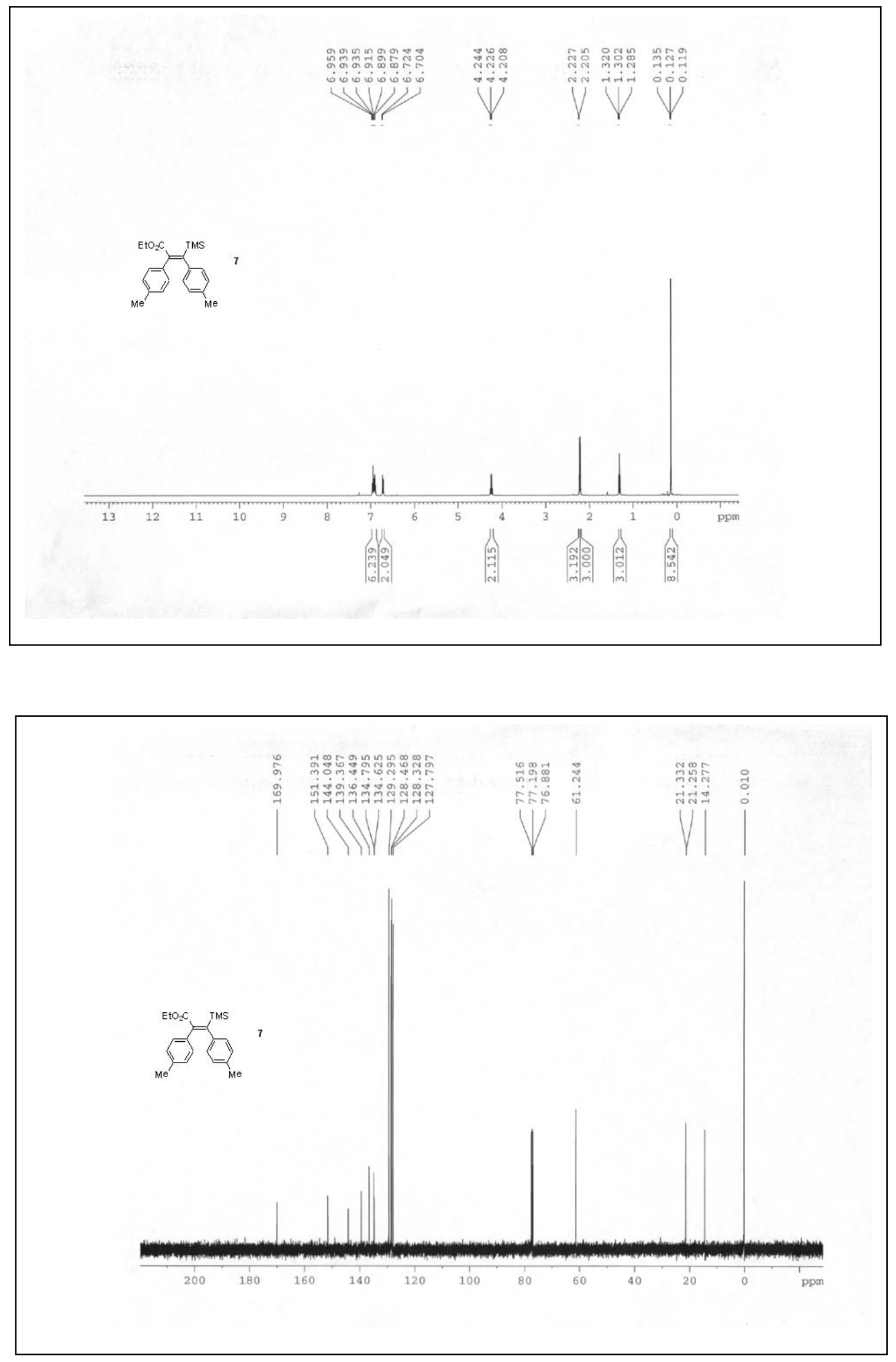

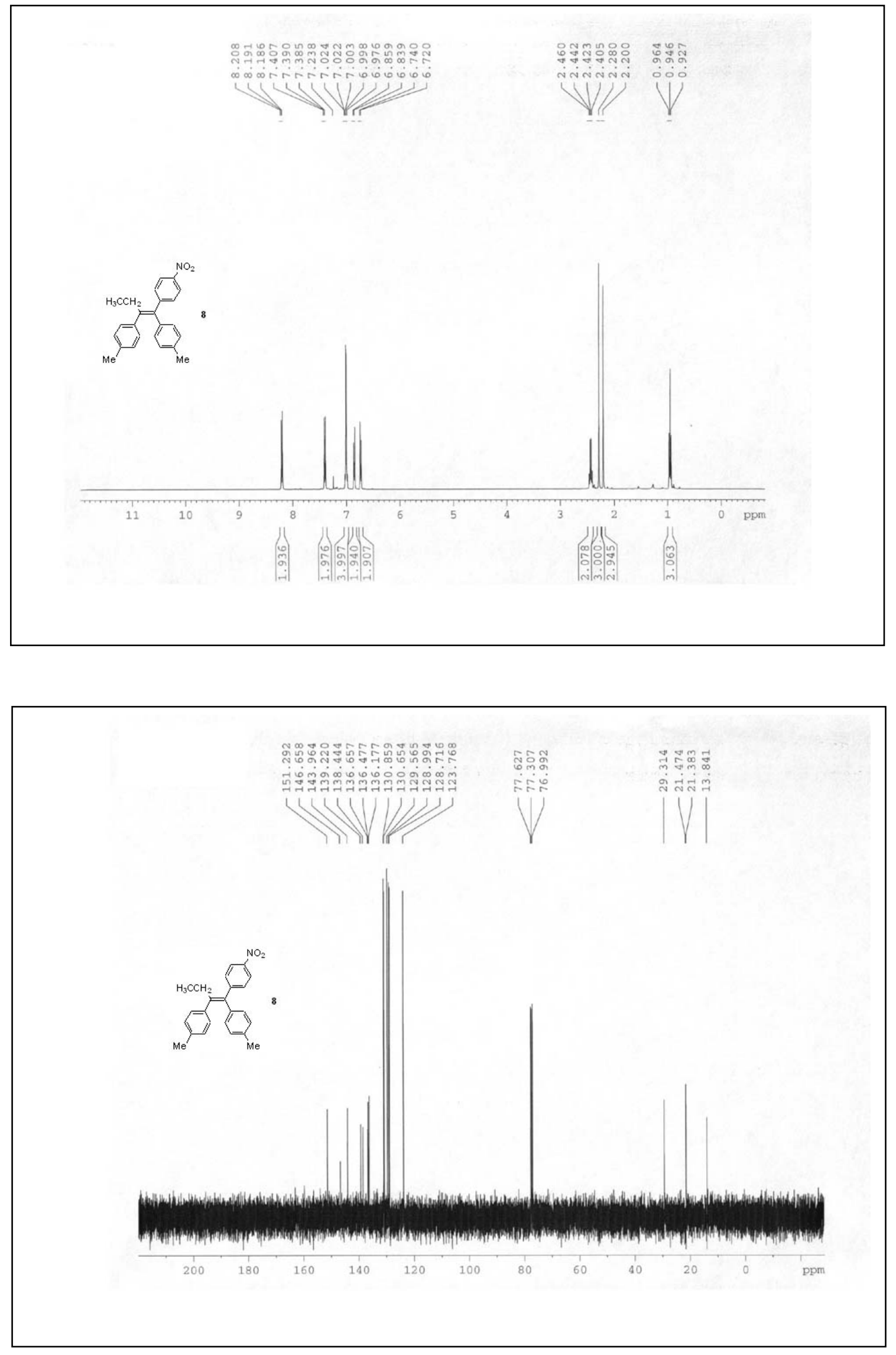

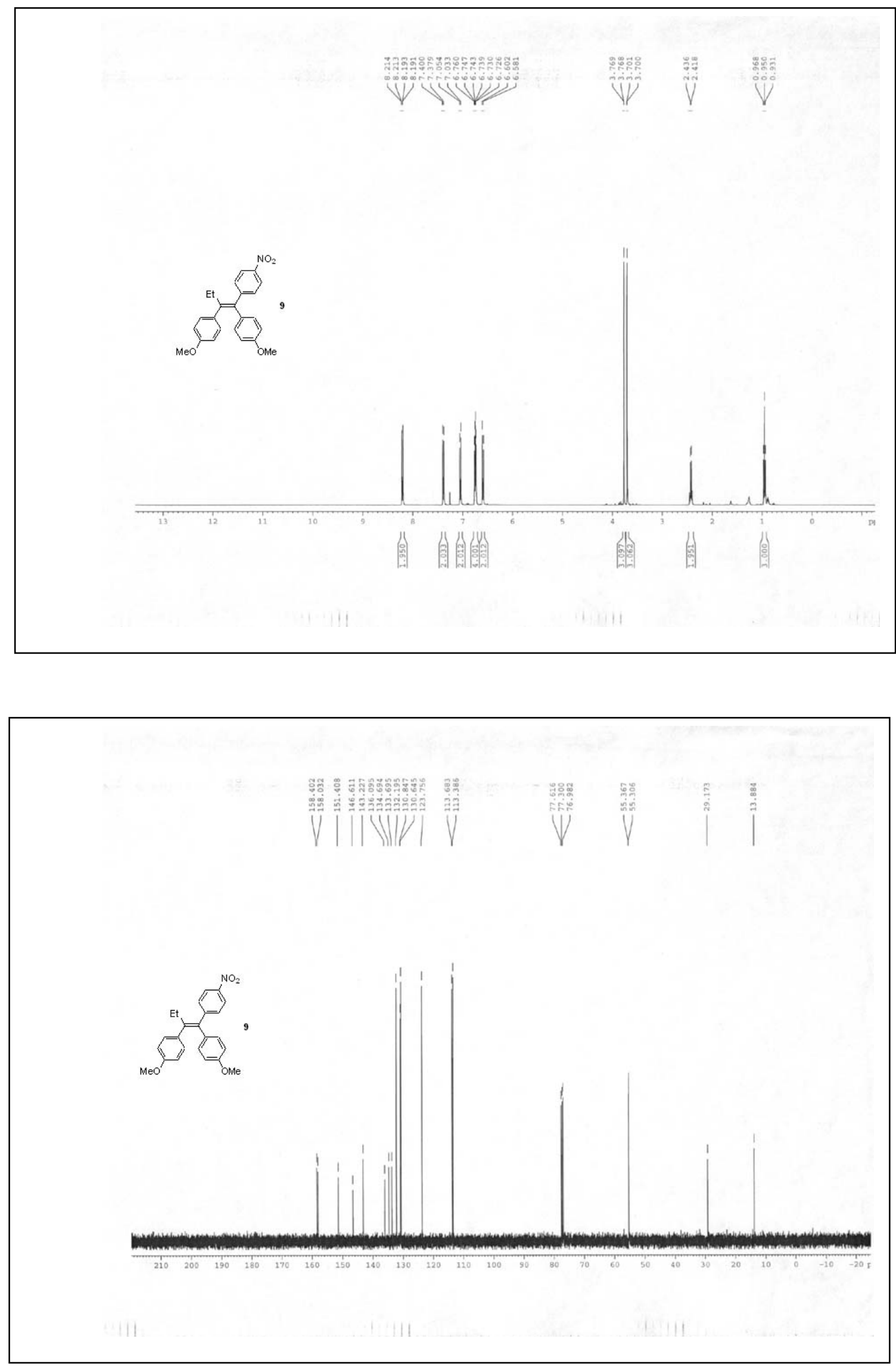

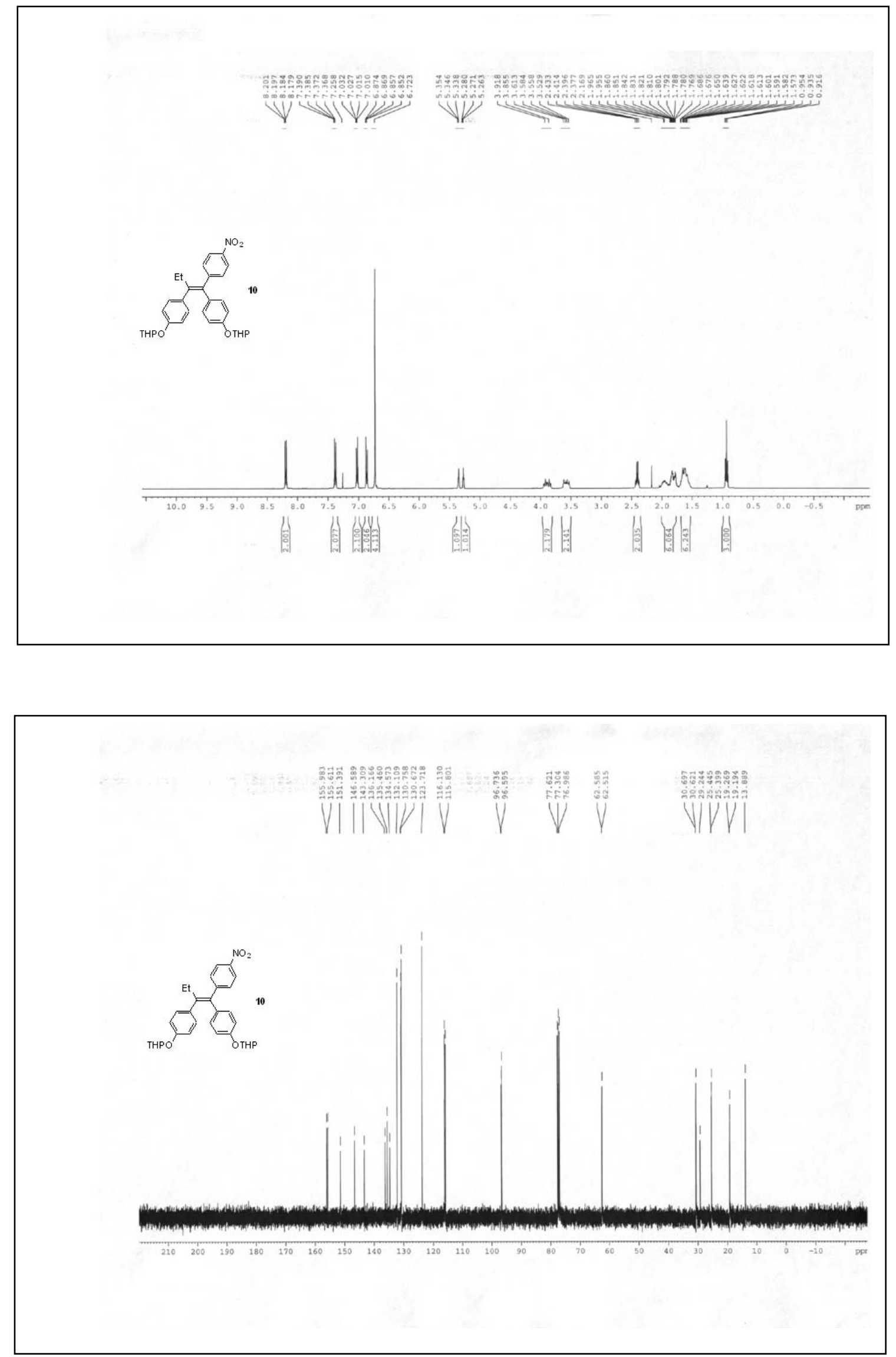

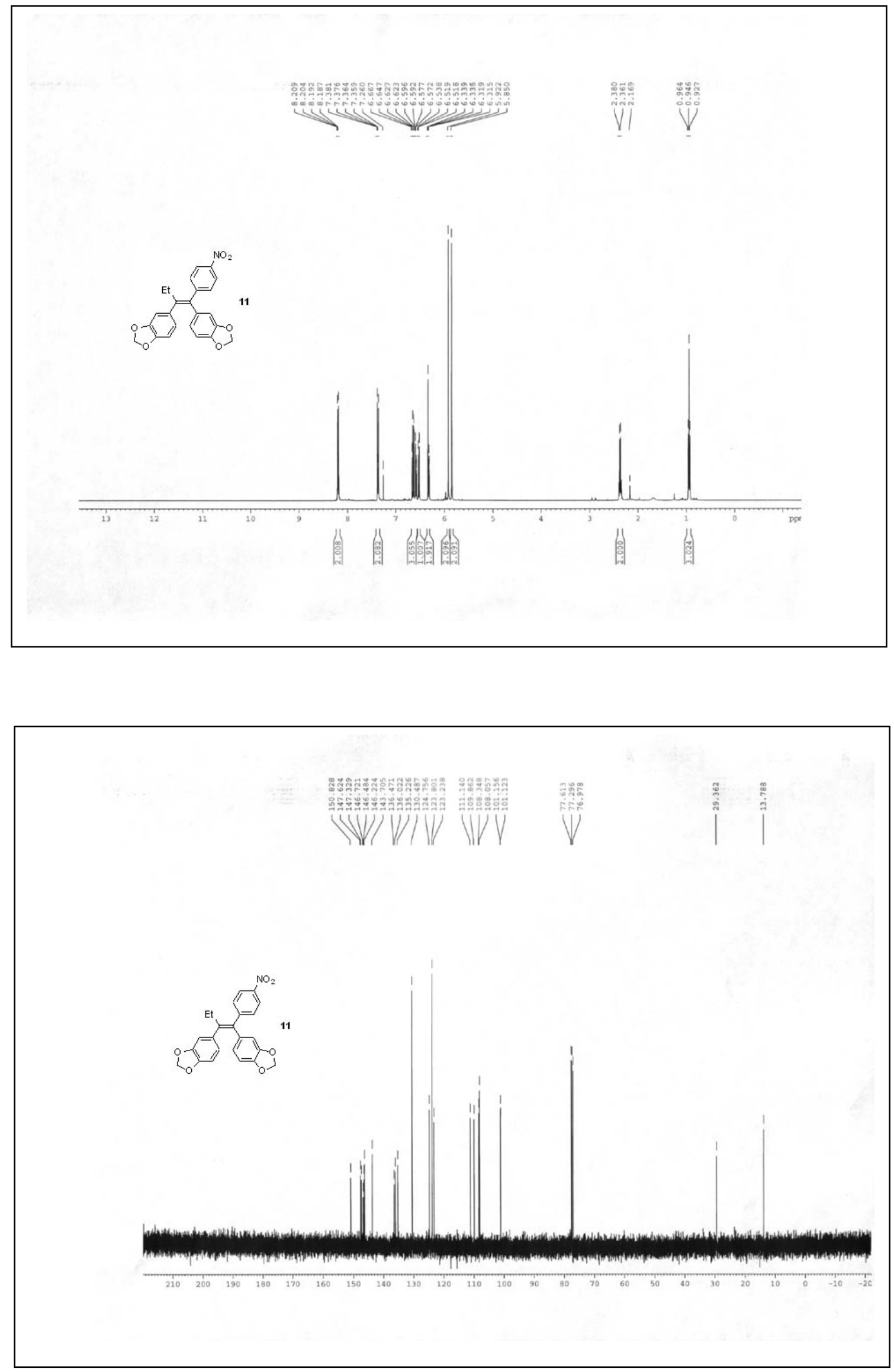

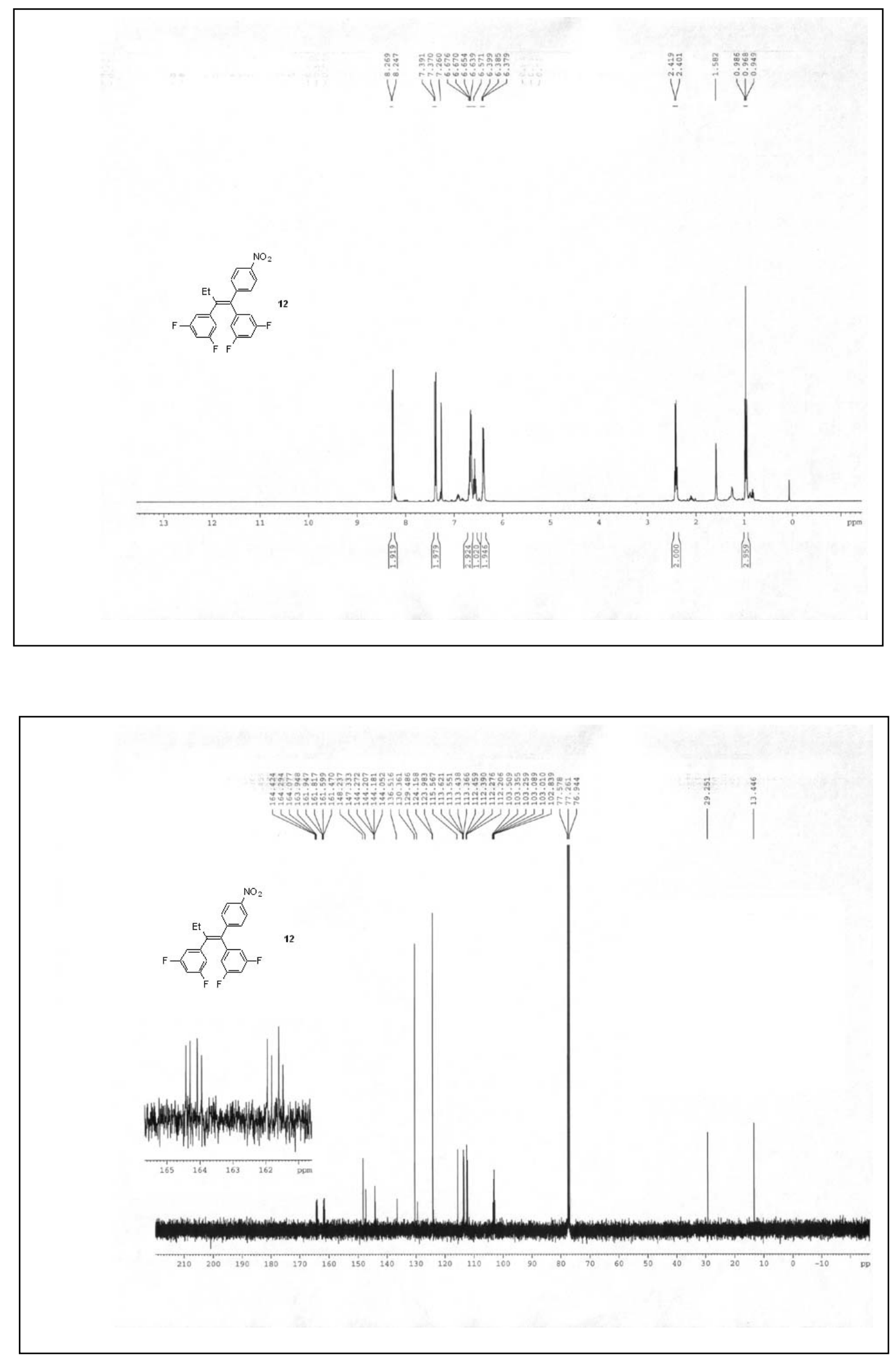

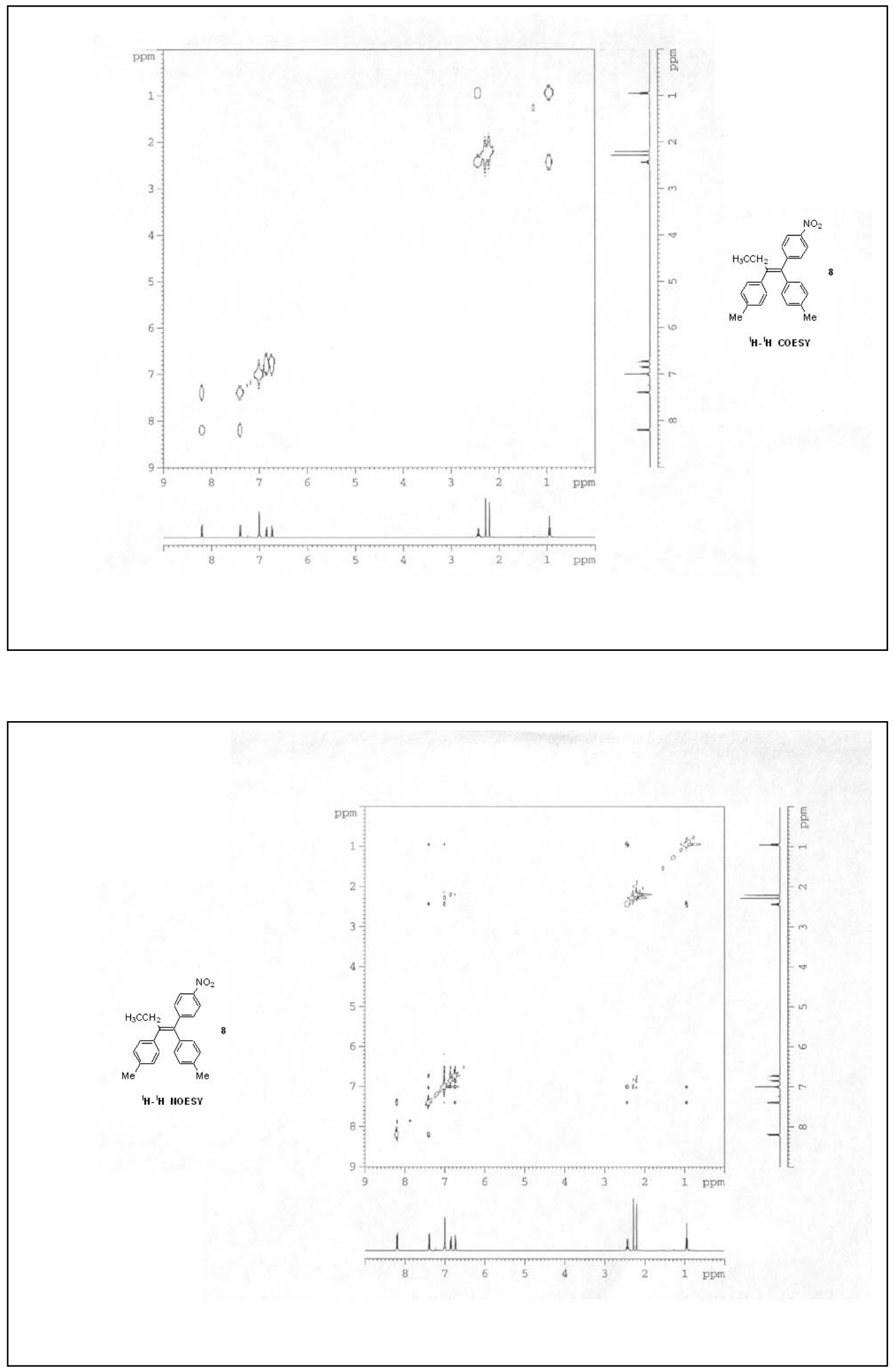